\title{
Design of Coplanar Reconfigurable Microwave Filter Design with Controllable Filtering Characteristics
}

\author{
Ayse Nihan Basmaci ${ }^{1}$, Karpuz Ceyhun ${ }^{2}$ \\ ${ }^{1}$ Corlu Vocational School, Namik Kemal University \\ Tekirdag, Turkey \\ anbasmaci@nku.edu.tr; ckarpuz@pau.edu.tr \\ ${ }^{2}$ Engineering Faculty, Pamukkale University \\ Denizli, Turkey
}

\section{Extended Abstract}

In this paper, interdigital capacitor loaded open loop square resonator and its single band reconfigurable filter applications are proposed. The proposed resonator is formed by a open loop square resonator having via connections. In addition, the square open loop resonator has interdigital capacitor located between the open ends.

The proposed resonator exhibits reconfigurable filtering characteristics with a small center frequency ratio. Resonance frequency can be controlled due to the changes in the interdigital capacitor and the electrical length of the resonator. Filtering characteristic of the band can be independently reconfigured bandpass to bandstop with the help of the via connection located between the open ends. By means of interdigital capacitor, small center frequency in the same physical area can be handled.

The designed resonator is analyzed by deriving even/odd mode resonance conditions. S-parameters are also calculated according to the even and odd mode input impedances. Passbands of the filter are adjusted to 1.7 and $2.7 \mathrm{GHz}$ which are allocated to mobile applications. Furthermore, position of the feedlines are used to control the in-band return loss levels. Theoretical analysis of the resonator is realized by defining the equivalent circuit model. Scattering and $\mathrm{ABCD}$ matrix of the designed filter are also extracted to clarify the filter topology.

A reconfigurable coplanar strip line filter is designed, simulated and analyzed theoretically. Simulation results and theoretically obtained results show a good agreement with the predicted results.

\section{References}

[1] M. F. Karim, Y. Guo, Z. Chen, and L. Ong, "Miniaturized reconfigurable filter using pin diode for UWB applications," in Proceedings of the IEE Microwave Symposium, pp. 1031-1034, 2008.

[2] M. F. Karim, A. Q. Lui, A. Alphones, and A. B. Yu, "A novel reconfigurable filter using periodic structures," in Proceedings of IEE MTT-S International Microwave Symposium, pp. 943-946, 2006.

[3] X. Huang, L. Zhu, Q. Feng, Q. Xiang and D. Jia, "Tunable bandpass filter with independently controllable dual passbands, "IEEE Transactions on Microwave Theory and Techniques, vol. 61, no. 9, pp. 3200-3208, 2013.

[4] M. F. Lei and H. Wang, "An analysis of miniaturized dual-mode bandpass filter structure using shunt-capacitance perturbation," in Microwave Theory and Techniques, IEEE Transactions, vol. 53, no. 3, pp. 861-867, 2005.

[5] A. Gorur, "Description of coupling between degenerate modes of a dual-mode microstrip loop resonator using a novel perturbation arrangement and its dual-mode bandpass filter applications," in IEEE Transactions Microwave Theory and Techniques, pp. 671-677, 2004. 